\title{
Qualidade de Vida, Apoio Social e Depressão em Idosos: Relação com Habilidades Sociais
}

\author{
Quality of Life, Social Support and Depression of the Elderly: \\ Relation with Social Abilities
}

\author{
Rachel Shimba Carneiro*a, Eliane Falcone ${ }^{\mathrm{a}}$, Cynthia Clark ${ }^{\mathrm{a}}$, \\ Zilda Del Prette ${ }^{b} \&$ Almir Del Prette ${ }^{b}$ \\ ${ }^{a}$ Universidade do Estado do Rio de Janeiro, Rio de Janeiro, Brasil \\ ${ }^{\mathrm{b}}$ Universidade Federal de São Carlos, São Carlo, Brasil
}

\begin{abstract}
Resumo
Estudos atuais apontam que a capacidade de interagir socialmente é fundamental para o idoso, a fim de que este possa conquistar e manter apoio social e garantir melhor qualidade de vida. O presente estudo teve como objetivo investigar as habilidades sociais, o apoio social, a qualidade de vida e a depressão de idosos da Universidade Aberta da Terceira Idade da Universidade do Estado do Rio de Janeiro (UnATI/UERJ), de contextos familiares e de asilos. Como instrumentos de pesquisa foram utilizados: o Inventário de Habilidades Sociais; a Medida de Apoio Social; o WHOQOL-ABREVIADO e a Escala de Depressão em Geriatria-15. A partir dos dados obtidos, observou-se que os idosos do asilo apresentaram um menor repertório de habilidades sociais, uma menor rede de apoio social e uma pior qualidade de vida. Essa pesquisa fornece subsídios para a implantação de Programas de Treinamentos de Habilidades Sociais específicos para terceira idade, contribuindo assim para que os idosos obtenham apoio social e uma melhor qualidade de vida.

Palavras-Chave: Habilidades sociais; qualidade de vida; apoio social; depressão; idosos.
\end{abstract}

\begin{abstract}
Current studies suggest that the capacity to socially interact is basic for the elderly in order to reach out and keep social support, and to guarantee a better quality of life. The present study aimed to investigate the social abilities, social support, quality of life and depression of the elderly from Universidade Aberta da Terceira Idade da Universidade do Estado do Rio de Janeiro (UnATI/UERJ), in the contexts of family and asylums. For this research the following tools were used: Social Skills Inventory, Social Support Measurement, WHOQOLBRIEF and Geriatric Depression Scale-15. Analysis of the data obtained from the instruments above, show that the elderly that live in asylums present a reduced repertoire of social abilities, a diminished social support network and an impaired quality of life. This research offers subsidies for the implantation of Social Skills Training Programs directed for the elderly, enhancing their ability to get social support and, therefore, a better quality of life.

Keywords: Social abilities; quality of life; social support; depression; elderly.
\end{abstract}

O crescimento da população de idosos é um acontecimento mundial e ocorre em um nível sem precedentes (Pereira, Curioni \& Veras, 2003). Em 1950, havia cerca de 204 milhões de idosos no mundo. Em 1998, menos de cinco décadas depois, esse contingente alcança 579 milhões de pessoas; um crescimento de quase oito milhões de idosos por ano (Instituto Brasileiro de Geografia e Estatística [IBGE], 2002). Esse fenômeno desperta atenção de pesquisadores, observando-se, também no Brasil, um crescente interesse pelas questões ligadas à qualidade de vida na terceira idade (Freire, 2000).

O reconhecimento de que a qualidade de vida constitui um tema importante para a promoção da saúde física e

\footnotetext{
* Endereço para correspondência: Rua Lopes Trovão, 134/1401, Bloco A, Bairro Icaraí, Niterói, Rio de Janeiro, RJ, 24220-071. E-mail rachelshimba@yahoo.com.br
} mental e para o bem-estar social das pessoas tem estimulado o Grupo de Qualidade de Vida da Organização Mundial de Saúde a realizar estudos envolvendo esse tema. A qualidade de vida foi definida por esse grupo como "a percepção do indivíduo de sua posição na vida, no contexto da cultura e sistema de valores nos quais ele vive e em relação aos seus objetivos, expectativas, padrões e preocupações" (Fleck et al., 2000, p. 179).

As investigações sobre as condições que permitem uma boa qualidade de vida na velhice revestem-se de grande importância científica e social (Fleck, Chachamovich \& Trentini, 2003). Por exemplo, Santos, Costa Santos, Fernandes e Henriques (2002), mostram que a qualidade de vida do idoso pode estar relacionada aos seguintes componentes: capacidade funcional, estado emocional, 
interação social, atividade intelectual e autoproteção de saúde. Já Frutuoso (1999) indica que muitos estudos fazem referência a um aumento da qualidade de vida e da longevidade em idosos que apresentam vida social intensa. Além disso, uma relação direta entre relacionamentos sociais, qualidade de vida e capacidade funcional e uma relação inversa desses fatores com a depressão têm sido apontadas por diversos autores (por ex., Albuquerque, 2003; Fleck et al., 2002; Xavier, Ferraz, Bertollucci, Poyares \& Moriguchi, 2001). Esses dados sustentam a importância dos relacionamentos sociais para o bem-estar físico e mental na velhice (Capitanini, 2000) e, conseqüentemente, para uma vida com qualidade.

$\mathrm{O}$ argumento em favor da idéia de que as relações sociais podem, de várias formas, promover melhores condições de saúde tem sido salientada em vários estudos (Ramos, 2002; Silberman et al., 1995). Por outro lado, a ausência de convívio social causa severos efeitos negativos na capacidade cognitiva geral (Katz \& Rubin, 2000), além de depressão (Freire \& Sommerhalder, 2000). A pobreza de relações sociais como um fator de risco à saúde tem sido considerada tão danosa quanto o fumo, a pressão arterial elevada, a obesidade e a ausência de atividade física (Andrade \& Vaitsman, 2002). Este conjunto de evidências sugere que a deterioração da saúde pode ser causada não somente por um desgaste natural do organismo, sedentarismo ou uso de tabaco, mas, também, pela redução da quantidade ou qualidade das relações sociais (Ramos, 2002). De uma forma geral, pode-se argumentar que as pessoas que têm maior contato social vivem mais e com melhor saúde do que as pessoas com menor contato social (Dressler, Balieiro \& Santos, 1997).

No entanto, Chelala (1992, citado por Oliveira, Pasian \& Jacquemin, 2001) ressalta que a qualidade dos contatos sociais é mais importante do que a quantidade. Como propõem Carmona e Melo (2000), uma pessoa que tem uma conduta interpessoal efetiva é capaz de desenvolver redes sociais de apoio, que operam como um dos fatores mais relevantes na recuperação e prevenção dos problemas de saúde mental. Em uma revisão feita por Chor, Griep, Lopes e Faerstein (2001), foi encontrado que o termo apoio social refere-se ao "grau em que relações interpessoais correspondem a determinadas funções (por exemplo, apoio emocional, material e afetivo) em situações de necessidade" (p. 888). Cobb (1976) pontua que o apoio social leva o indivíduo a acreditar que é querido, amado e estimado, e que faz parte de uma rede social com compromissos mútuos. Declarações desse tipo apontam para a necessidade de não se confundir apoio social com interação social, salientandose que nem todo relacionamento oferece apoio e que alguns relacionamentos podem ser, na realidade, muito estressantes (Dickson-Markman \& Shern, 1990, citado por Goldstein, 1995). Assim, parece que a capacidade de interagir socialmente é fundamental para o idoso, para conquistar e manter redes de apoio social e, conseqüentemente, garantir melhor qualidade de vida (Gray, Ventis \& Hayslip, 1992).

A constatação de que as habilidades sociais estão relacionadas a melhor qualidade de vida (Caballo, 1991; Collins \&
Collins, 1992; Goleman, 1995; Ickes, 1997) vem despertando o interesse de especialistas e multiplicando as pesquisas sobre o tema (Z. A. P. Del Prette \& A. Del Prette, 1999). Por outro lado, as deficiências e comprometimentos de habilidades sociais estão geralmente associados a dificuldades nas relações interpessoais e a diversos tipos de transtornos psicológicos como, por exemplo, a timidez, o isolamento social, o suicídio e a depressão (Z. A. P. Del Prette \& A. Del Prette, 1999, 2002). É importante, conforme Z. A. P. Del Prette e A. Del Prette (2001), diferenciar os termos habilidades sociais, competência social e desempenho social:

O desempenho social refere-se à emissão de um comportamento ou seqüência de comportamentos em uma situação qualquer. Já o termo habilidades sociais refere-se à existência de diferentes classes de comportamentos sociais no repertório do indivíduo para lidar de maneira adequada com as demandas das situações interpessoais. A competência social tem sentido avaliativo que remete aos efeitos do desempenho social nas situações vividas pelo indivíduo (p. 31 ).

Em estudo mais recente, esses autores chamam atenção para o uso cuidadoso desses conceitos. As habilidades sociais devem ser tomadas em um "sentido descritivo, de caracterização do repertório do indivíduo” (2005, p. 8). Dessa maneira, designar alguém como "socialmente habilidoso" não seria adequado, uma vez que "esse termo é mais avaliativo do que descritivo” (A. Del Prette \& Z. A. P. Del Prette, 2005, p. 8). A competência social, por sua vez, deve ser compreendida "sob uma perspectiva avaliativa a respeito da proficiência do desempenho” (A. Del Prette \& Z. A. P. Del Prette, 2005, p. 8). Assim, a competência social está relacionada à capacidade de "organizar pensamentos, sentimentos e ações em função de seus objetivos e valores, articulando-os em um desempenho que atende às demandas mediatas e imediatas do ambiente" (A. Del Prette \& Z. A. P. Del Prette, 2005, p. 8-9).

O conjunto das habilidades requeridas para relações interpessoais satisfatórias inclui diferentes classes e subclasses dentre as quais as habilidades de comunicação, assertivas, empáticas, de civilidade, de expressividade emocional, de trabalho etc. (Z. A. P. Del Prette \& A. Del Prette, 2001). Falcone (2000, 2001) destaca a importância das habilidades empáticas e de sua vinculação às assertivas para que as interações sociais sejam bem sucedidas. A empatia é entendida como a capacidade de compreender e de expressar compreensão acurada sobre a perspectiva e sentimentos de outra pessoa, além de experimentar compaixão e interesse pelo bem-estar desta (Barret-Lennard, 1993; Falcone, 1999). O conceito de comportamento socialmente competente deve incluir a capacidade do indivíduo para obter satisfação pessoal (assertividade) e, ao mesmo tempo, a motivação genuína para compreender e atender às necessidades da outra pessoa (empatia) (Falcone \& Ramos, 2005). A falta e/ou deficiência em manifestar empatia e assertividade pode ocasionar padrões de comportamento socialmente inadequados, tais como a esquiva ou a agressividade. Esses padrões acabam prejudicando a qualidade da interação e favorecendo os conflitos sociais.

O comportamento socialmente competente não compreende apenas desempenho aberto (verbais e não verbais) 
mas também componentes cognitivos de atenção, percepção e processamento de informação. Na interação social eficaz, o indivíduo seleciona, de forma acurada, informações úteis e relevantes de um contex to interpessoal, usa essa informação para apresentar comportamentos apropriados dirigidos a meta e, finalmente, executa os comportamentos verbais e não verbais que maximizam a probabilidade de obter e manter a meta de boas relações com os outros (Beddell \& Lennox, 1997; Carmona \& Melo, 2000).

Apesar de se reconhecer a importância do repertório social do idoso, a literatura especializada ainda é escassa em estudos de avaliação nessa área, podendo-se, aqui, destacar a pesquisa realizada por Carneiro e Falcone (2004), onde foi utilizada uma entrevista estruturada para investigar as situações nas quais 30 idosos, que freqüentavam uma universidade da terceira idade, apresentavam capacidades e deficiências em interagir de forma socialmente competente. As autoras concluíram que os idosos relataram habilidades em situações que facilitavam a aquisição de apoio social e que não envolviam risco de conflito. Por outro lado, estes apresentavam déficits naquelas situações que envolviam confronto interpessoal. Esses dados são concordantes com os estudos de Cavalcanti (1995), os quais apontam o treino afirmativo como uma das intervenções mais eficazes no atendimento psicoterápico à terceira idade. Considerando-se que os idosos dessa amostra já possuíam uma rede de apoio social, pode-se supor que aqueles que carecem desse apoio apresentem um grau maior de deficiências em habilidades sociais, especialmente as que envolvem comportamento assertivo.

Embora a literatura aponte uma associação entre relações interpessoais, apoio social e qualidade de vida na terceira idade, são escassas as pesquisas que focalizam, especificamente, o repertório de habilidades sociais de idosos em sua relação empírica com essas variáveis. Pode-se supor que o idoso que conta com uma rede de apoio social tende a ser mais socialmente competente, além de possuir níveis mais elevados de qualidade de vida do que aquele que interage apenas com o seu grupo familiar e com alguns amigos. Por outro lado, idosos saudáveis que vivem em asilos, por carecerem de apoio social, podem apresentar deficiências em habilidades sociais, além de baixa qualidade de vida e, conseqüentemente, níveis mais elevados de depressão.

Essas suposições estão na base dos objetivos desta pesquisa, que pretende comparar as relações entre habilidades sociais, apoio social, qualidade de vida e depressão em idosos de três diferentes grupos sociais: (a) os que possuem rede de apoio social, (b) os que não participam de atividades sociais fora do seu contex to familiar, e (c) os que moram em asilos.

\section{Método}

\section{Participantes}

A amostra consistiu de 75 idosos (63 do sexo feminino e 12 do sexo masculino, com idades variando de 61 a 95 anos), distribuídos da seguinte forma: 30 idosos que freqüentam ambientes de ensino, como a Universidade Aberta da Ter- ceira Idade da Universidade do Estado do Rio de Janeiro (GUF); 30 idosos residentes em seus lares não engajados em atividades sociais em instituições (GF) e 15 idosos que vivem em asilos (GA).

\section{Instrumentos e Material}

Cada participante recebeu o Termo de Consentimento Livre e Esclarecido (TCLE) contendo informações sobre os objetivos da pesquisa, a gravação das verbalizações emitidas nas situações de jogos de papéis e o compromisso com o sigilo a respeito da identidade dos participantes.

Uma Ficha de Entrevista foi utilizada para a obtenção de dados pessoais referentes à amostra estudada (idade, sexo, localização geográfica, estado civil, escolaridade, tempo de ingresso na Universidade Aberta ou tempo de residência no asilo).

Um instrumento de avaliação de qualidade de vida, elaborado pela Organização Mundial da Saúde (WHOQOLABREVIADO), traduzido e validado no Brasil por Fleck et al. (2000), foi utilizado na pesquisa. Tal instrumento consta de 26 itens, com duas questões gerais sobre qualidade de vida e as demais 24 divididas em quatro domínios: (a) físico, (b) psicológico, (c) relações sociais e (d) meio ambiente. As características psicométricas do WHOQOL-ABREVIADO preencheram os critérios de consistência interna, validade discriminante, validade concorrente, validade de conteúdo e confiabilidade teste-reteste (Fleck et al., 2000). É importante ressaltar que, como a maioria dos idosos do asilo não respondeu ao item 21 (“Quão satisfeito você está com a sua vida sexual?”), que faz parte do domínio social e ao item 25 ("Quão satisfeito você está com o seu meio de transporte?”), que se insere no domínio meio ambiente do WHOQOL-ABREVIADO, a análise dos dados foi realizada sem essas duas perguntas.

O Inventário de Habilidades Sociais (IHS-Del-Prette, de Z. A. P. Del Prette \& A. Del Prette, 2001), utilizado para avaliar as habilidades sociais dos participantes, é composto de 38 itens que se agrupam em cinco fatores:

1. Enfrentamento e auto-afirmação.

2. Auto-afirmação na expressão de sentimentos positivos.

3. Conversação e desenvoltura social.

4. Auto-exposição a desconhecidos e situações novas.

5. Autocontrole da agressividade.

O fator 1 avalia principalmente o conceito de assertividade; o fator 2 retrata demandas interpessoais de expressão de afeto positivo e de afirmação da auto-estima; o fator 3 apresenta situações sociais neutras de aproximação, demandando, principalmente, "traquejo social” na conversação, o que supõe conhecimento das normas de relacionamento cotidiano. As situações que envolvem a abordagem de pessoas desconhecidas são medidas pelo fator 4. Por fim, o fator 5 reúne itens que supõem reação a estimulações aversivas do interlocutor, demandando controle da raiva e da agressividade. Conforme consta no Manual, propriedades psicométricas do IHS-Del-Prette apresentam índices 
de validade, fidedignidade e consistência interna satisfatórios. À seguir, serão apresentados alguns itens do questionário que foram adaptados ao contex to de indivíduos da terceira idade: item 2. Quando um de meus familiares (filhos, irmão, sobrinhos ou cônjuge) ou um de meus amigos insiste em dizer o que eu devo fazer, contrariando o que penso, acabo aceitando para evitar problemas; item 5. Quando um(a) amigo(a) ou parente a quem emprestei dinheiro, esquece de me devolver, encontro um jeito de lembrá-lo(a); item 8. Mesmo junto a conhecidos, encontro dificuldade em participar da conversação; item 9. Se tiver que fazer um discurso ou uma palestra para um grupo de pessoas desconhecidas, eu evito; item 10. Expresso sentimentos de carinho através de palavras e gestos a meus familiares, amigos e colegas; item 11. Em uma reunião com várias pessoas, se uma delas faz uma afirmação incorreta, eu exponho meu ponto de vista; item 13. Se alguém me faz um elogio, fico encabulado(a) sem saber o que dizer; item 14. Caso seja solicitado, eu faço uma palestra para um grupo de pessoas; item 15. Quando um familiar ou pessoa próxima me critica injustamente, expresso-lhe diretamente meu aborrecimento; item 17. Em uma conversação com amigos, tenho dificuldade em encerrar a conversa, preferindo aguardar que outros o façam; item 18. Quando um de meus familiares ou um de meus amigos, por algum motivo, me critica, reajo de forma agressiva; item 22. Ao ser solicitado por um colega para dizer uma pequena mentira em favor dele acabo aceitando mesmo achando que não devia; item 29. Em um banco ou numa loja, quando não compreendo uma explicação de um funcionário sobre algo que estou interessado(a), faço as perguntas que julgo necessárias ao meu esclarecimento; item 34. Concordo em fazer as tarefas que me pedem e que não são da minha obrigação, mesmo sentindo um certo abuso nesses pedidos; item 38. Consigo "levar na esportiva" as gozações de amigos e parentes a meu respeito.

O apoio social foi avaliado por meio da Medida de Apoio Social de Chor et al. (2001), que contém cinco dimensões funcionais:

1. Material, que investiga a disponibilidade de pessoas para a provisão de recursos práticos e ajuda material.

2. Afetivo, que verifica a freqüência de demonstrações físicas de amor e afeto das pessoas da rede social do indivíduo.

3. Emocional, que avalia a freqüência de expressões de afeto positivo, compreensão e sentimentos de confiança das pessoas da rede social do indivíduo.

4. Interação social positiva, que investiga a disponibilidade de pessoas para se divertirem ou relaxarem junto.

5. Informação, que identifica a disponibilidade de pessoas para a obtenção de conselhos ou orientações. Os índices de consistência interna e de teste-reteste deste instrumento foram satisfatórios (Chor et al., 2001).

Além disso, foi utilizada a Escala de Depressão em Geriatria-15 (GDS-15) que foi validada e adaptada no Brasil por Almeida e Almeida (1999), sendo esta considerada apropriada para identificar um possível caso de depressão, es- pecificamente para pessoas na terceira idade. O GDS-15 contém uma lista de sintomas cujo resultado da quantidade está fortemente associado ao diagnóstico nosológico. A avaliação da consistência interna da escala indica que esta é uma medida válida para a deteç̧ão de casos de depressão entre idosos (Almeida \& Almeida, 1999).

Os dados de observação direta foram obtidos com base em testes comportamentais orientados por um roteiro que estruturava situações de jogos de papéis. As situações de jogos de papéis utilizadas foram:

1. Recusar pedido ("Você está a caminho para o consultório médico, quando encontra um amigo que não vê faz algum tempo. Ele dá um grande sorriso e, desconhecendo o seu compromisso, convida-o para tomar um café, para contar sobre a viagem que fez. O que você diria nessa situação social?").

2. Fazer pedido com conflito de interesses ("Você foi comprar pão no supermercado. Quando vai se dirigir ao caixa, verifica que as filas são longas e que as pessoas estão abarrotadas de compras. Se você ficar esperando sua vez, não conseguirá chegar a tempo do programa marcado com seu amigo. O que você faz?").

3. Defender os próprios direitos em situações nas quais são oferecidos serviços insatisfatórios ("Há duas semanas atrás, você comprou uma roupa nova numa loja da vizinhança que você tem sido cliente. $\mathrm{E}$ depois de vestir a roupa pela segunda vez, você descobriu que o zíper está quebrado. O que você faz? Caso volte na loja, como falaria com a vendedora?").

4. Lidar com pessoas com atitudes grosseiras ("Quando lhe pedem um favor, você está sempre pronta para ajudar e faz com boa vontade. Certo dia de manhã uma pessoa te pede para buscar uma roupa e você acabou esquecendo. No momento que essa pessoa fica sabendo do seu esquecimento, ela te chama de imprestável, e diz: não sei onde estava com a cabeça para te pedir isso. O que você responderia nessa situação?”).

5. Expressar opiniões pessoais ("Você está numa roda de amigos e colegas que estão falando de um filme que você achou ruim. Entretanto, as pessoas ao seu redor estão elogiando o filme e argumentando o quanto ele é bom. Num determinado momento perguntam o que você achou do filme. O que você responde?”).

\section{Procedimento de Coleta de Dados}

Após aprovação da Comissão de Ética em Pesquisa da Universidade do Estado do Rio de Janeiro (COEP/UERJ) e do Comitê de Ética em Pesquisa da Universidade Aberta da Terceira Idade da Universidade do Estado do Rio de Janeiro (UnATI/UERJ), foi feito um contato formal com a diretora de um asilo, visando o consentimento para a realização da presente pesquisa. Em seguida, os idosos que voluntariamente concordaram em participar receberam o TCLE. Após a assinatura deste documento, foi entregue a ficha de entrevista, seguida pela aplicação do IHS-Del- 
Prette e do WHOQOL-ABREVIADO. Posteriormente, o GDS- 15 e a Medida de Apoio Social foram entregues respectivamente. Depois de responder os quatro inventários de auto-informe, os participantes se submeteram às cinco situações de jogos de papéis. A aplicação das medidas de avaliação foi realizada individualmente e em um único encontro, além disso, não houve tempo pré-determinado para o preenchimento dos questionários utilizados e para responder às situações de jogos de papéis.

\section{Aspectos Éticos}

A elaboração desta pesquisa foi realizada buscando observar e atender as normas da Comissão de Ética em Pesquisa da Universidade do Estado do Rio de Janeiro - COEP/ UERJ e do Comitê de Ética em Pesquisa da UnATI/UERJ.

Todos os idosos que participaram do estudo foram informados do objetivo da pesquisa e da gravação das verbalizações emitidas nas situações de jogos de papéis, assim como foi explicado que a identidade deles e as verbalizações gravadas seriam mantidas em sigilo. Após esses esclarecimentos, cada participante era solicitado a ler o TCLE e assiná-lo, caso concordasse em colaborar com a pesquisa.

\section{Tratamento dos Dados}

Inicialmente, de acordo com as instruções normativas de cada instrumento, foram calculados os escores totais de cada respondente para o IHS-Del-Prette (Z. A. P. Del Prette \& A. Del Prette, 2001), o WHOQOL-ABREVIADO (Fleck et al., 2000) e a Medida de Apoio Social (Chor et al., 2001), bem como os escores fatoriais de cada participante e os valores médios por grupo em cada um dos fatores, domínios e dimensões, respectivamente. Para a comparação entre os grupos (GUF, GF e GA), foi usada a análise da variância simples (one-way ANOVA) com post-hoc(LSD), com o tipo de grupo entrando como variável independente e os quatro domínios do WHOQOL-ABREVIADO, os cinco fatores do IHS-DelPrette e as cinco dimensões funcionais da Medida de Apoio Social entrando como variáveis dependentes.

As distribuições das pontuações das variáveis utilizadas foram testadas através do teste de Kolmogorov-Smirnov para uma amostra, a fim de verificar se estas eram significativamente diferentes da normalidade. O nível de significância adotado para este teste estatístico, bem como para os demais, foi de $5 \%$. Como não foram encontrados desvios significativos da normalidade, adotamos testes estatísticos paramétricos para as comparações de médias. Para verificar a associação entre habilidades sociais, qualidade de vida e apoio social, foi calculado o coeficiente de correlação de Pearson.

Em relação às verbalizações emitidas nas cinco situações de jogos de papéis, estas foram gravadas e depois transcritas integralmente, sendo então avaliadas por dois juízes independentes, previamente treinados e cegos quanto aos grupos da amostra ao qual cada sujeito pertencia. Para orientar os juízes na avaliação das respostas dos participantes, foi construído um manual contendo explicações e exemplos de três categorias de avaliação, de acordo com a proposta de Falcone (2000, 2001): (a) respostas sociais inadequadas; (b) respostas sociais parcialmente adequadas e (c) respostas sociais adequadas. Os juízes foram orientados a enquadrar, na categoria número 1 , as respostas passivas (de esquiva) ou agressivas (de hostilidade). Na categoria 2, as respostas de enfrentamento da situação (assertivas), de defesa dos próprios interesses de forma objetiva, sem demonstrar claramente o interesse pelas necessidades, desejos ou sentimentos do interlocutor. Finalmente, na categoria 3, as respostas de enfrentamento que incluem a defesa dos próprios interesses, desejos, necessidades, após demonstração clara de compreensão e de interesse pelos desejos e necessidades do interlocutor (empatia e assertividade). Levando-se em conta que as verbalizações emitidas pelos participantes, em cada situação de jogo de papel, receberam de cada juiz uma única avaliação (resposta social inadequada, resposta social parcialmente adequada e resposta social adequada), foram calculados os coeficientes de concordância entre os dois juízes (Bauer, 2002). Os resultados indicaram um índice de concordância variando de $76 \%$ à $98 \%$. Para a obtenção de um consenso nos itens em que existiram discordâncias, foi marcado um novo encontro com os juízes.

\section{Resultados}

Os resultados referentes a cada um dos conjuntos de dados são apresentados a seguir. Inicialmente, a Tabela 1 mostra os resultados descritivos obtidos em cada um dos grupos para os domínios, fatores e dimensões dos três instrumentos utilizados.

Com base nos dados da Tabela 1, na próxima página, a comparação estatística entre as médias apresentadas, em cada um dos instrumentos, permitiu constatar uma diferença significativa, entre os três grupos, para os seguintes domínios do WHOQOL-ABREVIADO (Fleck et al., 2000): (1) físico (ANOVA: $\mathrm{F}(2,72)=6,2 ; p=0,003) ;(2)$ psicológico (ANOVA: $\mathrm{F}(2,72)=17,0 ; p=0,000$ ); (3) social (ANOVA: $\mathrm{F}(2,72)=4,2 ; p=0,019)$ e (4) meio ambiente (ANOVA: $\mathrm{F}(2,68)=27,1 ; p=0,000)$.

Comparando-se o GUF com o GA, as diferenças foram altamente significativas $(p<0,01)$ nos quatro domínios do WHOQOL-ABREVIADO (Fleck et al., 2000) com superioridade para o GUF. Comparando-se o GF com o GA, também foi constatada uma diferença altamente significativa ( $p<0,01)$, favorável ao GF nos domínios psicológico, social e meio ambiente. Já na comparação de GUF com GF, foi encontrada diferença significativa apenas no domínio psicológico $(p=0,019)$.

$\mathrm{Na}$ análise das duas questões gerais de qualidade de vida do WHOQOL-ABREVIADO verificou-se, com relação à satisfação com a saúde, que o GF $(3,27)$ apresentou a menor média quando comparado ao GA $(3,60)$ e ao GUF $(3,77)$. Na segunda pergunta, sobre a qualidade de vida, a maioria do GUF (57\%) e do GF (57\%) avaliaram como "boa", enquanto que boa parte dos participantes do GA (47\%) avaliou como "nem ruim nem boa".

Em relação às habilidades sociais, as médias apresentadas na Tabela 1 revelaram diferenças significativas na aná- 
Tabela 1

Valores Médios dos Escores Fatoriais do WHOQOL-ABREVIADO, do IHS-Del-Prette e da Medida de Apoio Social nos Grupos Estudados

\begin{tabular}{|c|c|c|c|c|}
\hline \multirow[t]{2}{*}{ Instrumentos } & \multirow[t]{2}{*}{ Fatores, domínios e dimensões } & \multicolumn{3}{|c|}{ Grupos } \\
\hline & & GUF & GF & GA \\
\hline \multirow[t]{4}{*}{ WHOQOL-ABREVIADO } & Domínio 1: físico & 3,93 & 3,60 & 3,20 \\
\hline & Domínio 2: psicológico & 4,21 & 3,93 & 3,32 \\
\hline & Domínio 3: meio ambiente & 3,74 & 3,71 & 2,72 \\
\hline & Domínio 4: relações sociais & 4,20 & 4,30 & 3,60 \\
\hline \multirow[t]{5}{*}{ IHS-Del-Prette } & Fator 1: enfrentamento e auto-afirmação com risco & 2,31 & 2,02 & 1,47 \\
\hline & Fator 2: auto-afirmação na expressão de sentimento positivo & 3,50 & 3,41 & 2,92 \\
\hline & Fator 3: conversação e desenvoltura social & 2,74 & 2,45 & 1,73 \\
\hline & Fator 4: auto-exposição a desconhecidos e situações novas & 1,98 & 1,53 & 0,67 \\
\hline & Fator 5: autocontrole da agressividade & 3,28 & 3,14 & 3,31 \\
\hline \multirow{5}{*}{ Medida de Apoio Social } & Dimensão 1: material & 4,00 & 4,34 & 4,00 \\
\hline & Dimensão 2: afetiva & 4,32 & 4,57 & 3,18 \\
\hline & Dimensão 3: emocional & 3,85 & 4,14 & 2,77 \\
\hline & Dimensão 4: interação social positiva & 3,97 & 4,15 & 2,72 \\
\hline & Dimensão 5: informação & 3,95 & 4,08 & 3,42 \\
\hline
\end{tabular}

lise da variância simples, entre os três grupos, para os seguintes fatores do IHS-Del-Prette: (a) enfrentamento e autoafirmação com risco $(\mathrm{F}(2,72)=6,5 ; p=0,003)$; (b) auto-afirmação na expressão de sentimentos positivos $(\mathrm{F}(2,72)=6,0$; $p=0,004) ;(\mathrm{c})$ conversação e desenvoltura social $(\mathrm{F}(2,72)=6,7 ; p=0,002)$ e (d) auto-exposição a desconhecidos e situações novas $(\mathrm{F}(2,72)=6,5 ; p=0,002)$. Apenas no fator relacionado ao autocontrole da agressividade não ocorreram diferenças significativas $(\mathrm{F}(2,72)=0,3 ; \mathrm{n} . \mathrm{s})$.

No post-hoc (LSD), ao se comparar o GUF com o GF, não foram encontradas diferenças significativas em nenhum dos cinco fatores do IHS-Del-Prette. Entretanto, comparando o GUF com o GA, foram encontradas diferenças altamente significativas, $(p<0,01)$ favoráveis ao GUF, em quatro dos cinco fatores: F1, F2, F3 e F4. A comparação entre os GF e GA revelou diferenças significativas nos mesmos fatores, favoráveis ao GF. Nas duas comparações, não se encontrou diferença significativa no F5.

Em relação ao apoio social, as médias apresentadas na Tabela 1 evidenciaram uma diferença significativa nas dimensões afetiva (ANOVA: $\mathrm{F}(2,72)=14,2 ; p=0,000$ ), emocional (ANOVA: $\mathrm{F}(2,72)=8,6 ; p=0,000$ ) e social (ANOVA: $\mathrm{F}(2,72)=10,7 ; p=0,000)$. Entretanto, não houve diferença significativa na dimensão material (ANOVA: $\mathrm{F}(2,71)=1,2$; $p=0,300)$ e nem na dimensão referente à informação (ANOVA: $\mathrm{F}(2,72)=1,9 ; p=0,158)$. A comparação entre as médias dos três grupos nas cinco dimensões da Medida de Apoio Social, revelou que o GUF e o GF apresentaram as maiores médias. Ao comparar os dados do GUF e do GA, observou-se diferenças significativas $(p<0,01)$ nas dimensões afetiva, emocional e social. Entretanto, não houve diferença significativa entre o GUF e o GA na dimensão material $(p=1,000)$ e informação $(p=0,126)$. Ao comparar o GUF e o GF, não foram constatadas diferenças significativas nas dimensões material $(p=0,156)$, afetiva $(p=0,265)$, emocio- nal $(p=0,290)$, social $(p=0,487)$ e de informação $(p=0,658)$. Por fim, ao comparar o GF com o GA, foram encontradas diferenças significativas $(p<0,01)$ nas dimensões afetiva emocional e social, embora tais diferenças não tenham ocorrido nas dimensões material $(p=0,256)$ e de informação $(p=0,060)$.

A seguir, a Tabela 2 mostra a porcentagem de respostas sociais inadequadas, parcialmente adequadas e adequadas dos três grupos, nas cinco situações de jogos de papéis, obtidos a partir da avaliação dos juízes.

A partir dos dados apresentados na Tabela 2, é possível observar alguns aspectos importantes nas situações de jogos de papéis, como por exemplo: (a) para todos os grupos, a situação mais "fácil", ou seja, com maior proporção de reações adequadas e menor de inadequadas foi a de defender os próprios direitos ("Estou retornando aqui para comunicar que o zíper da roupa que comprei está com defeito. Eu gostaria do conserto ou da troca"); (b) as mais difíceis foram as de fazer pedido com conflito de interesse ("Eu prefiro ficar na fila esperando e perder o programa do que fazer o pedido") e lidar com pessoas grosseiras ("Fico calada"), esta última com a quase totalidade dos participantes dos três grupos apresentando reações inadequadas; (c) GUF e GF apresentaram reações mais adequadas nas de recusar pedidos ("É um prazer encontrar você, só que eu não posso demorar porque estou com um compromisso. Agora realmente ficará um pouco difícil para aceitar o seu convite, porque eu vou ao médico. Mas, a gente pode marcar um dia para nos encontrarmos. Telefonarei para você”) e defender os próprios direitos e mais inadequadas na de lidar com pessoas grosseiras; (d) com exceção de expressar opiniões pessoais ("Eu não gostei do filme e ponto final. Gosto não se discute"), na qual se equiparou com os demais grupos, o GA apresentou maior proporção de reações inadequadas nas situações de jogos de papéis. 
A partir da Escala de Depressão em Geriatria-15, foi constatado que entre os participantes do GF, 30\% apresentaram possível caso de depressão, enquanto que, no GUF, a percentagem ficou em apenas 10\%. Já no GA verificou-se uma maior porcentagem, alcançando um total de 40\% dos sujeitos com depressão.

Considerando-se a relação entre as escalas, os resultados identificaram correlações estatisticamente significativas entre: os escores totais das variáveis habilidade social e apoio social ( $r=0,265, n=74, p=0,023)$, os escores totais da habilidade social e da qualidade de vida $(r=0,456, n=71, p=0,000)$ e os escores totais do apoio social e da qualidade de vida ( $r=0,416, n=71, p=0,000)$. Além disso, a partir dos resultados obtidos na Escala de Depressão em Geriatria-15 (Almeida \& Almeida, 1999), pode-se constatar uma correlação inversa entre a depressão e as variáveis habilidades sociais $(r=-0,335$, $n=75, p=0,003)$, apoio social $(r=-0,243, n=74, p=0,037) \mathrm{e}$ qualidade de vida $(r=-0,529, n=71, p=0,000)$. Esses dados sugerem que as habilidades sociais, o apoio social e a qualidade de vida estão, de alguma forma, interligados.

\section{Discussão}

Conforme os resultados do IHS-Del-Prette (Z. A. P. Del Prette \& A. Del Prette, 2001), observou-se que, de uma forma geral, a maioria dos participantes da amostra apresentou um maior repertório de habilidades sociais em situações que envolvem a auto-afirmação na expressão de sentimentos positivos (agradecer elogios; fazer elogios, expressar sentimentos positivos) e o autocontrole da agressividade (lidar com críticas; lidar com chacotas e cumprimentar desconhecidos). Por outro lado, a maior parte dos participantes apresentou um menor repertório de habilidades sociais nas situações de enfrentamento e auto-afirmação com risco (devolver à loja uma mercadoria defeituosa; discordar do gru- po; lidar com críticas injustas; fazer pergunta a conhecidos, declarar sentimento amoroso, discordar de autoridade; falar a público conhecido; cobrar dívida de amigo, manter conversa com desconhecidos, abordar para relacionamento sexual, apresentar-se a uma pessoa desconhecida) e nas de auto-exposição a desconhecidos e situações novas (fazer pergunta a desconhecido; pedir favores a desconhecidos; fazer apresentações ou palestras a um público desconhecido). Tais resultados são concordantes com o estudo realizado por Carneiro e Falcone (2004), em que os indivíduos idosos mostraram-se socialmente competentes nas situações sociais que não envolvem confronto, bem como com a proposição de Cavalcanti (1995), que aponta para a necessidade de treinamento assertivo em idosos.

Ao comparar os três grupos, é possível verificar que os idosos do asilo apresentaram um maior comprometimento nas habilidades sociais. Uma possível explicação para esses resultados refere-se ao isolamento em que os idosos residentes em instituições vivem, ou à pouca comunicação entre eles (Deps, 1993). É importante considerar que estudos sugerem que as deficiências e comprometimentos em habilidades sociais podem caracterizar relações sociais restritas e conflitivas (Z. A. P. Del Prette \& A. Del Prette, 1999, 2001).

Nas situações de jogos de papéis, a maioria dos indivíduos do GUF e do GF foi capaz de defender adequadamente os próprios direitos em situações como a de trocar uma mercadoria que está com defeito e a maior parte das pessoas do GUF apresentou respostas sociais adequadas para recusar pedido. Diante de tais constatações, é importante destacar que, segundo alguns autores (Neri, 1997; Nogueira, 2001), a participação em atividades educacionais, bem como a manutenção e o apoio das relações familiares favorecem o aumento do senso de auto-eficácia. Considerando-se que um funcionamento competente do indivíduo na interação com o

Tabela 2

Percentagem de Respostas Inadequadas, Parcialmente Adequadas e Adequadas dos Três Grupos, conforme Avaliação dos Juízes

\begin{tabular}{|c|c|c|c|c|}
\hline Habilidades avaliadas & Grupo & Inadequada & Parcial & Adequada \\
\hline \multirow[t]{3}{*}{ Recusar pedido } & GUF & $10 \%$ & $30 \%$ & $60 \%$ \\
\hline & GF & $10 \%$ & $47 \%$ & $43 \%$ \\
\hline & GA & $27 \%$ & $53 \%$ & $20 \%$ \\
\hline \multirow[t]{3}{*}{ Fazer pedido com conflito de interesses } & GUF & $74 \%$ & $23 \%$ & $3 \%$ \\
\hline & GF & $87 \%$ & $13 \%$ & o \% \\
\hline & GA & $100 \%$ & $0 \%$ & $0 \%$ \\
\hline \multirow[t]{3}{*}{ Defender os próprios direitos } & GUF & $33 \%$ & $10 \%$ & $57 \%$ \\
\hline & GF & $30 \%$ & $23 \%$ & $47 \%$ \\
\hline & GA & $40 \%$ & $20 \%$ & $40 \%$ \\
\hline \multirow[t]{3}{*}{ Lidar com pessoas com atitudes grosseiras } & GUF & $80 \%$ & $20 \%$ & $\mathrm{O} \%$ \\
\hline & GF & $86 \%$ & $7 \%$ & $7 \%$ \\
\hline & GA & $93 \%$ & $7 \%$ & $0 \%$ \\
\hline \multirow[t]{3}{*}{ Expressar opiniões pessoais } & GUF & $20 \%$ & $70 \%$ & $10 \%$ \\
\hline & $\mathrm{GF}$ & $37 \%$ & $56 \%$ & $7 \%$ \\
\hline & GA & $33 \%$ & $60 \%$ & $7 \%$ \\
\hline
\end{tabular}


ambiente não é só uma questão de saber "o que fazer" (Carmona \& Melo, 2000), mas envolve também crenças sobre a sua auto-eficácia, é possível sugerir que tais crenças podem estar contribuindo para que os idosos do GUF e do GF apresentem uma maior capacidade para defender seus próprios direitos e recusar um pedido de forma adequada socialmente do que os idosos do asilo. Além disso, enfrentar estas situações de forma habilidosa certamente contribui para o senso de auto-eficácia do indivíduo e, conseqüentemente, para a melhor qualidade de vida (Carneiro \& Falcone, 2004).

Os dados destacados até o presente momento parecem indicar que os participantes do GUF e do GF possuem uma maior capacidade para se comunicar socialmente do que os do GA. Levando-se em conta que diversos autores (Caballo, 1991; Collins \& Collins, 1992; Goleman, 1995; Ickes, 1997) têm relacionado as habilidades sociais a uma melhor qualidade de vida, parece possível supor que os idosos do GUF e do GF apresentem uma melhor qualidade de vida do que os do GA. De acordo com os estudos de Sousa, Galante e Figueiredo (2003), viver em asilo torna os idosos menos capazes de manter a qualidade de vida. Neri (2001) acrescenta que a manutenção de relações sociais com o cônjuge, com os familiares e, principalmente, com amigos da mesma geração favorece o bem-estar psicológico e social dos idosos.

É interessante observar que, em relação ao apoio material e de informação, o GUF o GF e o GA não apresentaram diferenças significativas. Os resultados parecem indicar que os idosos do asilo têm ajuda material suficiente e obtém orientação de forma paternalista, mas são carentes de apoio afetivo, emocional e de interação social positiva quando comparados aos idosos dos outros dois grupos. Esses achados estão em consonância com os estudos desenvolvidos por Deps (1993) e com as afirmações de Neri e Sommerhalder (2001), ao mostrarem que as instituições basicamente só levam em conta as necessidades biológicas dos indivíduos, esquecendo as necessidades de assistência sócio-cultural.

Ao comparar os três grupos do estudo, pôde-se observar que o grupo do asilo apresentou a maior percentagem de idosos com depressão. Tais dados são concordantes com revisão de estudos de Merlin, Baptista e Baptista (2004), na qual enfatizam que a vida em uma instituição está amplamente relacionada ao aumento da vulnerabilidade do idoso à depressão (Donohue, Acierno, Hasselt \& Hersen, 1995) e ao prejuízo na qualidade de vida (Fleck et al., 2002).

Concluindo, o presente estudo confirmou as relações existentes entre habilidades sociais, apoio social e qualidade de vida na terceira idade. Os idosos dos GUF e GF apresentaram níveis significativamente mais elevados de habilidades sociais, de apoio social e de qualidade de vida do que os idosos do grupo do asilo, os quais, por sua vez, apresentaram níveis significativamente mais elevados de depressão. Tais resultados fortalecem os estudos que sugerem ser as habilidades sociais um componente importante para a qualidade de vida e a saúde dos idosos. Da mesma maneira, as deficiências em habilidades sociais parecem constituir um fator de vulnerabilidade para a baixa qualidade de vida e para a depressão em indivíduos da terceira idade. Entretanto, é importante chamar atenção para o tamanho da amostra, que não permite fazer generalizações precipitadas. Algumas propostas sugeridas para futuros estudos na área incluem aumento do número de sujeitos da amostra, melhor controle de variáveis sócio-demográfica e avaliação do impacto das habilidades sociais e do apoio social na redução da depressão e, conseqüentemente, no aumento da qualidade de vida do idoso.

\section{Referências}

Albuquerque, S. M. R. L. de (2003). Qualidade de vida do idoso. São Paulo, SP: Casa do Psicólogo.

Almeida, O. P., \& Almeida, S. A. (1999). Confiabilidade da versão brasileira da escala de depressão em Geriatria (GDS): Versão reduzida. Arquivos de Neuro-Psiquiatria, 57(2), 421-426.

Andrade, G. B. de, \& Vaitsman, J. (2002). Apoio social e redes: Conectando solidariedade e saúde. Ciência da Saúde Coletiva, 7(4), 925-934.

Barrett-Lennard, G. T. (1993). The phases and focus of empathy. The British Psychological Society, 3-13.

Bauer, M. W. (2002). Análise de conteúdo clássica: Uma revisão. In M. W. Bauer \& G. Gaskell (Ed.), Pesquisa qualitativa com texto, imagem e som (pp. 189-217). Petrópolis, RJ: Vozes.

Beddell, J. R., \& Lennox, S. S. (1997). Handbook of communication and problem-solving sills training: A cognitive-behavioral approach. New York: John Wiley \& Sons.

Caballo, V. E. (1991). El entrenamiento en habilidades sociales. In V. E. Caballo (Ed.), Manual de técnicas de terapia y modificación de conducta (pp. 403-471). Madrid, España: Siglo Veintiuno.

Capitanini, M. E. S. (2000). Solidão na velhice: Realidade ou mito? In A. L. Neri \& S. A. Freire (Eds.), E por falar em boa velhice (pp. 69-89). Campinas, SP: Papirus.

Carmona, C. G. H., \& Melo, N. A. (2000). Comunicacion interpersonal: Programa de Entrenamiento en Habilidades Sociales. Santiago, Chile: Ediciones Universidad Católica de Chile.

Carneiro, R. S., \& Falcone, E. O. (2004). Um estudo das capacidades e deficiências em habilidades sociais na terceira idade. Psicologia em Estudo, 9(1), 119-126.

Cavalcanti, M. B. (1995). Idosos. In B. Rangé (Ed.), Psicoterapia comportamental (pp. 149-158). Rio de Janeiro, RJ: Psy.

Chor, D., Griep, R. H., Lopes, C. S., \& Faerstein, E. (2001). Social network and social support measures from the pró-saúde study: Pre-tests and pilot study. Cadernos de Saúde Pública, $17(4), 887-896$.

Cobb, S. (1976). Social support as a moderator of life stress. Psychosomatic Medicine, 38, 300-314.

Collins, J., \& Collins, M. (1992). Social skills training and the professional helper. New York: Willey.

Del Prette, A., \& Del Prette Z. A. P. (2005). Perguntas (im)pertinentes sobre a área do treinamento das habilidades sociais. In H. J. Guilhardi \& N. C. Aguirre (Eds.), Sobre comportamento e cognição: Expondo a variabilidade (pp. 5-13). Santo André, SP: ESETEC.

Del Prette, Z. A. P., \& Del Prette, A. (1999). Psicologia das habilidades sociais: Terapia e educação. Petrópolis, RJ: Vozes.

Del Prette, Z. A. P., \& Del Prette, A. (2001). Inventário de habilidades sociais: Manual de aplicação, apuração e interpretação. São Paulo, SP: Casa do Psicólogo.

Del Prette, Z. A. P., \& Del Prette, A. (2002). Psicologia das relações interpessoais. Petrópolis, RJ: Vozes. 
Deps, V. L. (1993). A ocupação do tempo livre sob a ótica de idosos residentes em instituições: Análise de uma experiência. In A. L. Neri (Ed.), Qualidade de vida e idade madura (pp. 191211). Campinas, SP: Papirus.

Donohue, B., Acierno, R., Hasselt, V. B. V., \& Hersen, M. (1995). Social skills training in a depressed, visually impaired older adult. Journal of Behavior Therapy \& Experimental Psychiatry, 26(1), 65-75

Dressler, W. W., Balieiro, M. C., \& Santos, J. E. (1997). The cultural construction of social support in Brazil: Associations with health outcomes. Culture, Medicine and Psychiatry, 21, 303335.

Falcone, E. (1999). A avaliação de um programa de treinamento da empatia com universitários. Revista Brasileira de Terapia Comportamental e Cognitiva, 1, 23-32.

Falcone, E. (2000). Habilidades sociais: Para além da assertividade. In R.C. Wielenska (Ed.), Sobre comportamento e cognição: Questionando e ampliando a teoria e as intervenções clínicas e em outros contextos (Vol. 6, pp. 211-221). São Paulo, SP: SET.

Falcone, E. (2001). Uma proposta de um sistema de classificação das habilidades sociais. In H. J. Guilhardi; M. B. B. P. Madi; P. P. Queiroz \& M. C. Scoz (Eds.), Sobre comportamento e cognição: Expondo a variabilidade (pp.195-209). Santo André, SP: ESETEC.

Falcone, E. \& Ramos, D.M. (2005). A tribuição como componente cognitivo das habilidades sociais e seu impacto na satisfação conjugal. In H. J. Guilhardi \& N. C. Aguirre (Eds.), Sobre comportamento e cognição: Expondo a variabilidade (pp. 182-191). Santo André, SP: ESETEC.

Fleck, M. P. A., Louzada, S., Xavier, M., Chachamovich, E., Vieira, G., Santos, L., \& Pinzon, V. (2000). Aplicação da versão em português do instrumento abreviado de avaliação da qualidade de vida "WHOQOL-bref". Revista Saúde Pública, 34(2), 178183

Fleck, M. P. A., Lima, A. F. B. S., Louzada, S., Schestasky, G., Henriques, A., Borges, V. R., Camey, S., \& Grupo Lido. (2002). Associação entre sintomas depressivos e funcionamento social em cuidados primários à saúde. Revista Saúde Pública, 36(4), 431-438.

Fleck, M. P. A., Chachamovich, E., \& Trentini, C. M. (2003). Projeto WHOQOL-OLD: Método e resultados de grupos focais no Brasil. Revista Saúde Pública, 37(6), 793-799.

Freire, S. A. (2000). Envelhecimento bem-sucedido e bem-estar psicológico. In A. L. Neri \& S. A. Freire (Eds.), E por falar em boa velhice (pp. 21-31). Campinas, RS: Papirus.

Freire, S. A., \& Sommerhalder, C. (2000). Envelhecer nos tempos modernos. In A. L. Neri \& S. A. Freire (Eds.), E por falar em boa velhice (pp. 125-135). Campinas, SP: Papirus.

Frutuoso, D. (1999). A terceira idade na universidade. Rio de Janeiro, RJ: Ágora da Ilha.

Goldstein, L. L. (1995). Stress e coping. Na vida adulta e na velhice. In A. L. Neri (Ed.), Psicologia do envelhecimento (pp. 145194). Campinas, SP: Papirus.

Goleman, D. (1995). Inteligência emocional(M. Santarrita, Trad.). Rio de Janeiro, RJ: Objetiva.

Gray, G. R., Ventis, D. G., \& Hayslip, B. (1992). Socio-cognitive skills as a determinant of life satisfaction in aged persons.
International Journal of Aging and Human Development, 35(3), 205-218.

Ickes, W. (1997). Introduction. In W. Ickes (Ed.), Empathic accuracy (pp.1-15). New York: The Guilford Press.

Instituto Brasileiro de Geografia e Estatística. (2002). Perfil dos idosos responsáveis pelos domicílios no Brasil 2000. Rio de Janeiro, RJ: Autor.

Katz, L., \& Rubin, M. (2000). Mantenha o seu cérebro vivo. São Paulo, SP: Sextante.

Merlin, M. S., Baptista, A. S. D., \& Baptista, M. N. (2004). Depressão e suicídio na terceira idade. In M. N. Baptista. Suicídio e depressão: Atualizações (pp. 195-2 16). Rio de Janeiro, RJ: Guanabara Koogan.

Neri, A. L. (1997). Qualidade de vida na velhice. In M. Delitti (Ed.), Sobre comportamento e cognição. A prática da análise do comportamento e da terapia cognitivo-comportamental (pp. 34-40). Campinas, SP: Arbytes.

Neri, A. L. (2001). Velhice e qualidade de vida na mulher. In A. L. Neri (Ed.), Desenvolvimento e envelhecimento (pp. 161-200). Campinas, SP: Papirus.

Neri, A. L., \& Sommerhalder, C. (2001). As várias faces do cuidado e do bem-estar do cuidador. In A. L. Neri (Ed.), Cuidar de idosos no contexto da família: Questões psicológicas e sociais (pp. 9-62). Campinas, SP: Alínea.

Nogueira, E. J. (2001). Rede de relações sociais: Um estudo transversal com homens e mulheres pertencentes a três grupos etários. Tese de Doutorado não-publicada, Universidade Estadual de Campinas, SP.

Oliveira, E. A. de, Pasian, S. R., \& Jacquemin, A. (2001). A vivência afetiva em idosos. Psicologia Ciência e Profissão, 21(1), 68-83.

Pereira, R. S., Curioni, C. C., \& Veras, R. (2003). Perfil demográfico da população idosa no Brasil e no Rio de Janeiro em 2002 Textos sobre Envelhecimento, 6(1), 43-59.

Ramos, M. P. (2002). Apoio social e saúde entre idosos. Sociologias, 7, 156-175.

Santos, S. R., Costa Santos, I. B., Fernandes, M. G. M., \& Henriques, M. E. R. M. (2002). Qualidade de vida do idoso na comunidade: Aplicação da Escala de Flanagan. Revista LatinoAmericana de Enfermagem, 1O(6), 757-764.

Silberman, C., Souza, C., Wilhems, F., Kipper, L., Wu, V., Diogo, C., Schmitz, M., Stein, A., \& Chaves, M. (1995). Cognitive deficit and depressive symptoms in a community group of elderly people: A preliminary study. Revista de Saúde Pública, 29(6), 444-450.

Sousa, L., Galante, H., \& Figueiredo, D. (2003). Qualidade de vida e bem-estar dos idosos: Um estudo exploratório na população portuguesa. Revista Saúde Pública, 37(3), 364-371.

Xavier, F. M. F., Ferraz, M. P. T., Bertollucci, P., Poyares, D., \& Moriguchi, E. H. (2001). Episódio depressivo maior, prevalência e impacto sobre qualidade de vida, sono e cognição em octogenários. Revista Brasileira de Psiquiatria, 23(2), 62-70.

Recebido: 20/12/2005 $1^{a}$ revisão: 19/04/2006 $2^{a}$ revisão: 15/09/2006 Aceite final: 26/09/2006 